\title{
A Dual Tree Complex Discrete Cosine Harmonic Wavelet Transform (ADCHWT) and Its Application to Signal/Image Denoising
}

\section{Shivamurti, S. V. Narasimhan}

Digital Signal Processing and Systems Group Aerospace Electronic and Systems Division, National Aerospace Laboratories, Bangalore, India.

Email: \{narasim, shivmurti\}@nal.res.in

Received March 23 ${ }^{\text {rd }}, 2011$; revised April 30 ${ }^{\text {th }}, 2011$; accepted May $11^{\text {th }}, 2011$.

\begin{abstract}
A new simple and efficient dual tree analytic wavelet transform based on Discrete Cosine Harmonic Wavelet Transform DCHWT (ADCHWT) has been proposed and is applied for signal and image denoising. The analytic DCHWT has been realized by applying DCHWT to the original signal and its Hilbert transform. The shift invariance and the envelope extraction properties of the ADCHWT have been found to be very effective in denoising speech and image signals, compared to that of DCHWT.
\end{abstract}

Keywords: Analytic Discrete Cosine Harmonic Wavelet Transform, Analytic Wavelet Transform, Dual Tree Complex Wavelet Transform, DCT, Shift Invariant Wavelet Transform, Wavelet Transform Denoising

\section{Introduction}

The wavelet transform (WT) provides signal compression, denoising and many more desirable processing features. In spite of these advantages, the WT suffers from many major problems. The WT coefficients oscillate about the zero value around the singularities. This will reduce the magnitude of WT coefficients near singularity where their values are expected to be large, making the singularity extraction and signal modeling difficult. Further, the WT is shift variant, i.e., around singularities, even for a small shift in input signal, there will be a large variation in the energy distribution among WT coefficients at different scales resulting in different WT patterns which have to be considered for further processing [1]. This is due to aliasing caused by decimation at each wavelet level. Such a shift variant nature of WT not only affects detection of transients but also denoising as signal is reconstructed by decimated modified samples resulting in strong glitches [2]. Also at low signal to noise ratio like below $0 \mathrm{~dB}$, the conventional denoising fails. However the signal compression achieved by WT is not affected by its shift variant property.

The WT is realized by a perfect reconstruction filter bank which involves analysis filter bank, down sampling, interpolation and synthesis filter bank. Here the aliasing caused by the use of non-ideal filters is cancelled by the synthesis filter bank. However the reconstructed signal by such a filter bank is highly sensitive to coefficient errors and may get affected severely. Further such a WT suffers from poor directional selectivity for diagonal features. The WT filters being real, separable and their frequency response symmetric about zero in four quadrants in the $2 \mathrm{D}$ frequency space, cannot distinguish between two opposing diagonal directions, i.e. $+45^{\circ}$ and $-45^{\circ}$ edge orientations $[3,4]$.

The undecimated WT solves the shift variance with additional computational load. However the directionality problem remains unsolved as undecimated WT cannot distinguish the two opposing diagonals as it uses separable filters. This blindness to such a directionality makes the processing and modeling of image features like ridges and edges difficult. For separable filters to have proper directionality, their frequency responses should be asymmetric for positive and negative frequencies and can be achieved by using complex wavelet filters [4]. However the difficulty involved in the design of complex filters satisfying perfect reconstruction prohibits their use in image processing.

The sinusoids in the Fourier transform in higher dimensions provide highly directional plane waves. The FT 
magnitude does not oscillate and provides a smooth envelope. Further the FT magnitude is shift invariant and also it does not suffer from aliasing and the signal reconstruction (inverse FT) does not involve any critical reconstruction criterion. These benefits are due to its complex exponential basis instead of real basis of WT. Thus realization of complex/analytic WT has become important. This approach has been applied to image segmentation, classification, deconvolution, image sharpening, motion estimation, coding, water marking [4,5], texture analysis and synthesis, seismic imaging and extraction of evoked potentials in EEG [6]. The analytic WT (AWT) has the features of both WT and FT and is appropriate for time-frequency analysis like STFT [7].

The complex/analytic WT been realized using dual tree filter bank structure and Hilbert transform (HT). Many variations of this structure have been achieved with various degrees of advantages and limitations. The absence of negative frequencies for an analytic signal not only reduces aliasing but also accounts for the decimation by a factor of 2 at each DWT stage resulting in shift invariance. But the global nature of HT (infinite both time and frequency extent) converts the finite support wavelet function to that of an infinite support and this makes the shift invariance and alias free nature as approximate. The WT realized by a two channel perfect reconstruction filter bank is computationally expensive and complicated due to explicit decimation, interpolation, associated filtering and involved delay compensation in reconstruction.

The harmonic wavelet transform (HWT) proposed by Newland [8] is simple and does not require explicit decimation, interpolation and associated filtering. The decimated components are achieved in the frequency domain by taking the inverse transform of each group of FT coefficients. The signal reconstruction is achieved by the inverse FT of properly concatenated FT coefficient groups. Here also, the WT coefficients become complex due to lack of DFT real symmetry. Further, the DFT values will be generally affected by the leakage due to signal truncation resulting in poor quality signal decomposition. To overcome these, the DCT based harmonic wavelet transform (DCHWT) has been proposed $[9,10]$. The real nature and the symmetrical signal extension of DCT respectively result in a real and more exact WT coefficients as the leakage is very much reduced [11]. The DCHWT has been used for signal/image compression and spectral estimation both with computational and performance advantage. For speech and image signals, the compression provided an adaptive wavelet packet algorithm based on DCHWT has been found to be not only better than that by DCHWT but also that by adap- tive Daubechies-2 wavelet packet. Further it has been used for efficient and accurate signal decomposition to overcome the cross-terms in Wigner-Ville time frequency distribution Also the DCTHWT has been extended to realize its shift invariant version. [12] which reduces the glitches when applied for denoising.

In the present work, a new dual tree analytic wavelet transform based on DCHWT (ADCHWT) has been proposed and is applied for signal and image denoising. The analytic DCHWT has been realized by applying DCHWT to the original signal and its HT. The shift invariance property of the ADCHWT has been illustrated and its contribution in association with its envelope extraction property has been found to be very effective in denoising compared to that of DCHWT [13].

\section{Dual Tree Analytic Discrete Cosine Harmonic Wavelet Transform (ADCHWT)}

In this section, the discrete cosine harmonic wavelet transform and the development of the new dual tree analytic discrete cosine harmonic wavelet transform (ADCHWT) will be considered.

\subsection{Discrete Cosine Harmonic Wavelet Transform (DCHWT)}

The filter bank realization of WT, involves down sampling of the components obtained by the individual bandpass filters. The restoration of the processed signal corresponding to overall spectrum at the original sampling rate, involves summation of the components after their upsampling and image rejection filtering. The harmonic wavelet transform based on DFT (DFHWT) realizes the subband decomposition in the frequency domain by grouping the Fourier transform (FT) coefficients and the inverse of these groups results in decimated signals. Further after processing, the FT of the subband signals can be repositioned in their corresponding positions to recover the overall spectrum, with the original sampling rate. Therefore, this will not involve explicit decimation and interpolation operations. As a consequence, no band limiting and image rejection filters are necessary. Also, while reconstruction, there are no delay compensations as the subband groups are synthesized in frequency domain by repositioning them. In view of this, the harmonic subband decomposition is very attractive due to its simplicity. However in the DFHWT, the grouping of the DFT coefficients with lack of conjugate symmetry makes the WT coefficients complex. For reconstruction after concatenation of the groups, the conjugate symmetry is restored to get the real signal.

The DFHWT is very attractive as long as no processing of the components is involved prior to inverse trans- 
formation. However, for a signal segment obtained without using any window function, there can be a severe leakage effect from one subband of the signal into another. If different subbands have to be processed differently, this is not achieved as the signal energy from one to another has already leaked. The DFHWT may be tolerable for a signal with well-separated frequency components of sufficiently high magnitude. But for closely spaced components of significantly different magnitudes, during the computation of the FT itself, the energy will leak from the higher amplitude component to the lower one (and vice versa). This results in a large bias in the spectral magnitude and may even totally eclipse smaller amplitude spectral peaks. In such a case, decomposing the signal based on DFHWT and processing the subbands may not be very effective. Further leakage in DFHWT will also limit its use in signal or image compression application. The reason for this is that it is not possible to get a good signal reconstruction by omitting the lower scales (corresponding to high frequencies) in WT as the leaked energy cannot be recovered unless all the scales are considered. Therefore to utilize the attractive features of the harmonic wavelet transform, DCT is used instead of DFT, which has a comparatively reduced leakage effect. This is due to symmetrical data extension which results in a smooth transition from one DCT period to the other without any discontinuity.

The wavelet transform $W_{x}(a, b)$ characterizes the correlation or similarity between the signal $x(t)$ to be analyzed and the wavelet function $\psi((t-b) / a)$. Such a correlation is given by

$$
W_{x}(a, b)=\frac{1}{a^{1 / 2}} \int_{-\infty}^{\infty} x(t) \psi^{*}\left(\frac{t-b}{a}\right) \mathrm{d} t
$$

where $\psi(t)$ is the prototype/mother wavelet. By shifting and scaling $\psi(t)$ by the parameters $b$ and $a$, respectively; all the basis functions

$\psi_{a, b}(t)=a^{-1 / 2} \psi((t-b) / a)$ are obtained. Equation (1) can be realized in the frequency domain using Parseval's theorem as

$$
W_{x}(a, b)=\frac{a^{1 / 2}}{2 \pi} \int_{-\infty}^{\infty} X(\omega) \Psi^{*}(a \omega) e^{j \omega b} \mathrm{~d} \omega
$$

Therefore the, the wavelet transform can be derived by windowing the spectrum $X(\omega)$ with $\Psi^{*}(a \omega)$ and inverse Fourier transforming the product.

$$
W_{x}(a, b)=a^{1 / 2} F^{-1}\left[X(\omega) \Psi^{*}(a \omega)\right]
$$

$\Psi(\omega)$ and $X(\omega)$ are the FT of the mother wavelet $\psi(t)$ and the signal $x(t)$. That is, $W_{x}(a, b)$ for a particular scale ' $a$ ' can be computed by the Equation (4b) using $X(\omega)$ and $\Psi(a \omega)$ by FFT algorithm.

For a real symmetric signal $x_{S}(t)$ and a real symmetric wavelet $\psi_{S}(t)$ function, Equation (2a) becomes [9]

$$
C_{x}(a, b)=\frac{a^{1 / 2}}{2 \pi} \int_{-\infty}^{\infty} X_{s}(\omega) \Psi_{s}(a \omega) \cos (\omega b) \mathrm{d} \omega
$$

$x_{S}(\omega)$ and $\Psi_{S}(\omega)$ are the Fourier transform of $x_{S}(t)$ and $\psi_{S}(t)$ respectively. (Generally the wavelet function is a symmetrical one but to have consistency in the notation $\psi_{S}(t)$ is used). In other words, they are the cosine transforms of $x_{S}(t)$ and the mother wavelet $\psi_{S}(t) . C_{x}(a, b)$ is the wavelet transform in cosine domain instead of Fourier domain. Hence the corresponding equation for Equation ( $2 b$ ) is

$$
C_{x}(a, b)=a^{1 / 2} C^{-1}\left[X_{s}(\omega) \Psi_{s}(a \omega)\right]
$$

Therefore the cosine wavelet transform coefficient $C_{x}(a, b)$ for a particular scale " $a$ " can be computed by the Equation (3b) using $X_{s}(\omega)$ and $\Psi_{s}(a \omega)$ by a fast cosine transform algorithm which indirectly uses FFT algorithm. $\Psi_{s}(\omega)$ is very simple for the Harmonic cosine wavelet transform (CHWT), and it is zero at all frequencies except constant over a small frequency band.

$$
\Psi_{s}(\omega)= \begin{cases}1, & \omega_{0}-\omega_{c}<\omega<\omega_{0}+\omega_{c}, \\ & -\omega_{0}-\omega_{c}<\omega<-\omega_{0}+\omega_{c}, \\ 0, & \text { otherwise }\end{cases}
$$

For this the wavelet $\psi_{S}(t)$ is,

$$
\psi_{S}(t)=\frac{\omega_{c}}{\pi} \frac{\sin \omega_{c} t}{\omega_{c} t} \cos \omega_{0} t
$$

$$
\begin{aligned}
& \text { Representing } \frac{\sin \omega_{c} t}{\omega_{c} t} \text { by } \sin c\left(\omega_{c} t\right), \\
& \psi_{S}(t)=\frac{\omega_{c}}{\pi} \cos \omega_{0} t \sin c\left(\omega_{c} t\right)
\end{aligned}
$$

Hence the mother wavelet is a cosine modulated sinc function. Here the decomposition of the signal in the frequency domain is simple but suffers from the problem of poor time localization due to slow decaying of the sinc function. Though a spectral weighing other than rectangular improves the localization in time it results in a non-orthogonal wavelet set. The type of spectral weighing will determine the wavelet as it is the cosine transform of the wavelet.

For the cosine harmonic wavelet transform, the spectral weighing is a symmetrical rectangular function and for a discrete signal it is zero except over symmetrical 
finite bands $[\pi / p, \pi / q]$ and $[-\pi / p,-\pi / q]$ where $p, q$ can be real numbers, not necessarily integers.

For an orthogonal CHWT, the wavelet function is fixed and corresponds to a rectangular weighing in the frequency domain which results in such a wavelet transform.

The Discrete cosine transform (DCT) enables the implementation of the above cosine transform discussed as it forms the symmetric signals $x_{S}(t)$ and $\psi_{S}(t)$ by itself (for the given non-symmetric $x(t)$ and $\psi(t)$ ). For a sampled signal $x(n), n=0,1,2, \cdots,(N-1)$, the DCT of $N$ points, is defined as the DFT of a $2 N$ point symmetrically extended signal $y(n)$.

$$
y(n)= \begin{cases}x(n), & 0 \leq n \leq N-1 \\ x(2 N-1-n), & N \leq n \leq 2 N-1\end{cases}
$$

$y(n)$ is even symmetric with respect to the $[N-(1 / 2)]$ point . This leads to DCT and is given by

$$
C_{x}(k)= \begin{cases}\sum_{n=0}^{N-1} 2 x(n) \cos \frac{\pi k(2 n+1)}{2 N}, & 0 \leq k \leq N-1 \\ -C_{x}(2 N-k), & N \leq k \leq 2 N-1\end{cases}
$$

Using the above $\Psi_{S}(\omega)$ in the CHWT, the subband decomposition is done in frequency domain unlike in time domain by a filter bank. This is achieved by grouping the $2 \mathrm{~N}$ coefficients of a discrete cosine transform (DCT) of length $2 N$ and this is equivalent to applying a window or weighing by a constant in the frequency domain.

The DCT coefficients can be grouped in a way similar to that of DFT coefficients and the DCT being real, there is no necessity to do the conjugate operation in placing the coefficients symmetrically [9]. The symmetrical placement is also not necessary due to the very definition of the DCT as it provides only half the number of coefficients and the inverse DCT definition takes care of the symmetry. The grouped coefficients for each band have to be treated as if they are the DCT coefficients of that subband.

In the Figure 1 DCTHWT for a DCT size of 16 is illustrated and the only one side of the symmetrical coefficient sequence is shown, i.e. $(0-7)$. The last half part of the coefficients $C_{x}(4)$ to $C_{x}(7)$ correspond to scale0 , i.e., $C_{0}$. The lower half of the coefficients $C_{x}(0)$ to $C_{x}(3)$ are split into two groups and the upper group $\left[C_{x}(2), C_{x}(3)\right]$ correspond to scale-1, i.e., $C_{1}$.

Further the group $\left[C_{x}(0), C_{x}(1)\right]$ is split into two groups $C_{2}$ and $C_{3}$ each having single coefficients $C_{x}(1)$ and $C_{x}(0)$, respectively. The inverse DCT of each of the groups $C_{0}, C_{1}, C_{2}, C_{3}$; result in WT coefficients of the scales $0,1,2,3$, respectively. For $Q=32$,

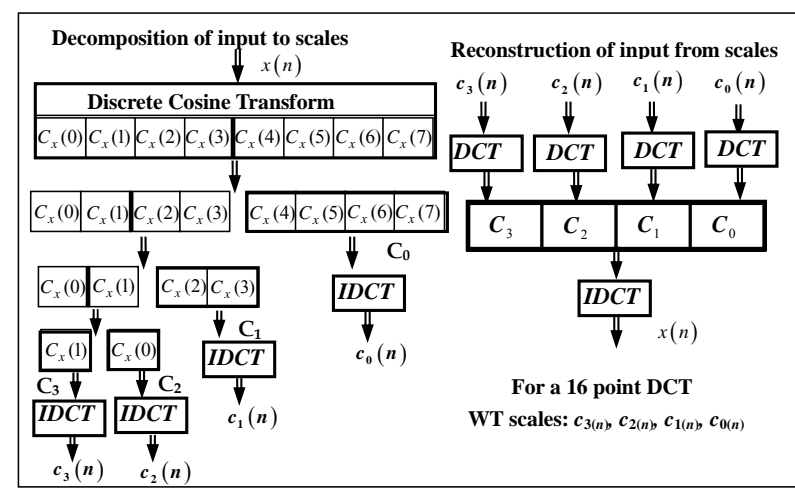

Figure 1. DCHWT for a 1-D signal.

the scales $C_{0}, C_{1}, C_{2}, C_{3}, C_{4}$ correspond to grouping of coefficients as $\left\{C_{x}(8)\right.$ to $\left.C_{x}(15)\right\},\left\{C_{x}(4)\right.$ to $\left.C_{x}(7)\right\}$, $\left\{C_{x}(2)\right.$ to $\left.C_{x}(3)\right\},\left\{C_{x}(1)\right\},\left\{C_{x}(0)\right\}$, respectively and this process continues.

For the reconstruction, the DCTs of the subband signals are concatenated to get the DCT of the fullband signal. For the first stage of inverse DCHWT illustrated in Figure 1, the DCTs of the subband signals corresponding to groups $\mathrm{C} 3$ and $\mathrm{C} 4$ are concatenated. The resulting group of coefficients is concatenated with the DCT of subband signal corresponding to group $\mathrm{C} 2$, in the next stage. Again, the resulting group of coefficients is concatenated with the DCT of subband signal corresponding to group $\mathrm{C} 1$, to form the DCT of the fullband signal.

\subsection{Dual Tree Analytic Discrete Cosine Harmonic Wavelet Transform (ADCHWT)}

There are different methods of obtaining the AWT, which uses the DWT. The straight forward post filtering approach splits each filter bank output into positive and negative frequency components using a complex PRFB acting as a HT though looks simple suffers from nonzero values in the negative frequency region.

The dual tree complex wavelet transform uses two DWT trees one for the real part of the analytic signal and the other for its imaginary part, the HT of the input. The corresponding scales of the two trees are combined to achieve the desired analytic WT. This does not require any complex filters and suffer from any performance limitation but its computational load is twice that of a DWT as two DWT trees. The proposed ACHWT is also realized by a dual tree approach and is shown in the Figure 2 for 4 scales. Here the DCHWT 4 scales for the original signal are obtained in the method explained in Subsection 2.1. The input signal is converted to its HT and again its DCHWT 4 scales are obtained. Thus the different scales and their HTs are obtained by simply 


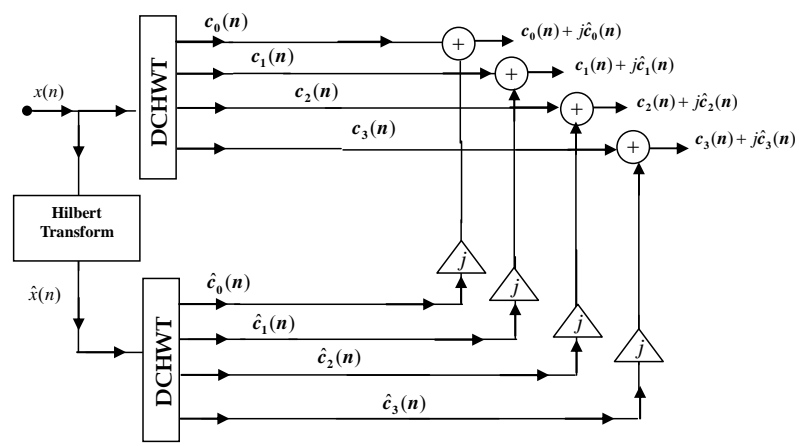

Figure 2. Schematic of the dual tree ADCHWT for a 1D signal.

converting the input to its HT. Further, Hilbert transformed scales are weighted by $j$ and are combined with their corresponding scales by summation to get the analytic harmonic discrete cosine wavelet transform (ADCHWT). For reconstruction, the real part of the ADCHWT is taken and the procedure is same as given in Subsection 2.1.

The HT forms an integral part of the ADCHWT and hence its quality determines the performance of $\mathrm{AD}$ CHWT. The analytic signal can be realized in the frequency domain by making the negative frequency components of the original signal to zero and scaling the positive components by a factor 2 and taking its inverse FT. The imaginary part of this analytic signal gives the desired HT of the signal. However, this method suffers from leakage problem of DFT as the energy from the negative frequency components would have leaked into the positive frequency region. Therefore, it is desirable to realize HT the signal in time domain by convolving the HT impulse response with the input signal [10]. The impulse response $h(n)$ of the HT is given by

$$
h(n)=\frac{2}{\pi}\left[\frac{\sin ^{2}(\pi n / 2)}{n}\right]-\infty \leq n \leq \infty
$$

In practice the length of the impulse response is same as data length. Hence truncating the impulse response results in Gibbs ripple effect in the frequency response of the HT. This Gibbs ripple will introduce distortion due to variation in gain in the passband of the signal. To overcome this, the HT impulse response has to be windowed by a smoothing window like Kaiser with an appropriate smoothing factor.

The shift invariance performance for the proposed ADCHWT is illustrated for two types of signals viz., an impulse and a rectangular pulse. The magnitude difference of the WT coefficients (for different scales) between original signal and its shifted version are plotted (Figure 3).

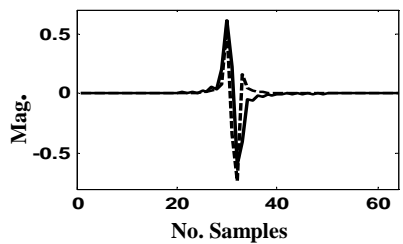

(a)

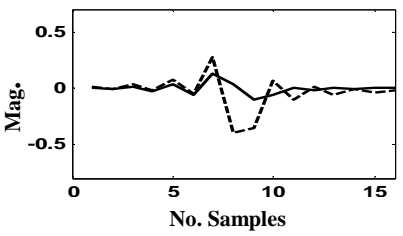

(c)

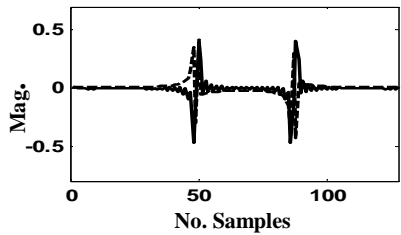

(e)

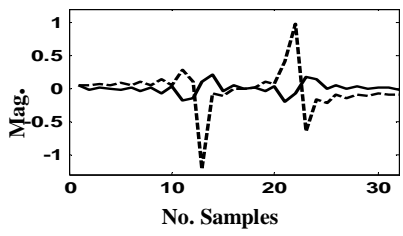

(g)

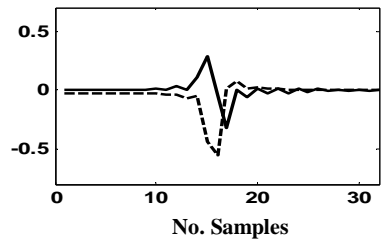

(b)

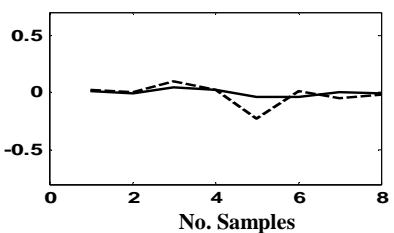

(d)

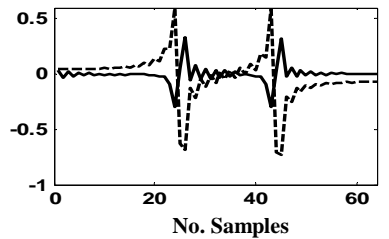

(f)

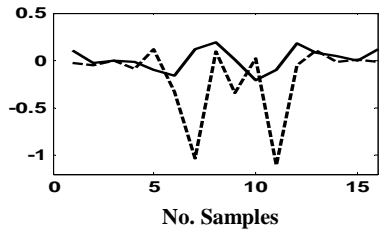

(h)
Figure 3. Difference in WT indicating energy for Impulse and pulse. For impulse: (a) Scale-0, (b) Scale-1, (c) Scale-2 (d) Scale-3 For pulse: (e) Scale-0, (f) Scale-1 , (g )Scale-2, (h) Scale-3 - - DCHWT, ADCHWT.

It is seen that for the higher scales the magnitude of the difference between WT coefficients for the original and shifted inputs (by 4 samples) is higher for the DCHWT than for ADCHWT both for the impulse and pulse inputs. Also the energy of this WT difference is indicated in Table 1. It is seen that with the ADCHWT, this difference energy is significantly small compared to that of DCHWT as the latter gets affected by the shift due to its shift variant nature.

\subsection{Two Dimensional Dual Tree ADCHWT}

For a 2D signal, the DCT coefficients for the columns are split in to two groups and their inverse DCT results in DCTHWT coefficients for the columns. The DCT along the rows for each scale are taken and grouped. The inverse DCT of these groups will result in 2D DCTHWT (Figure 4(a)). This procedure is repeated for further scales considering the LL block as input. The procedure holds good for the real part of ADCHWT of an image.

For the imaginary part of ADCHWT image on hand, 
Table 1. Error energy between the WT of original and shifted signal.

\begin{tabular}{ccccc}
\hline WT Type & \multicolumn{2}{c}{ DCHWT } & \multicolumn{2}{c}{ ADCHWT } \\
\hline Scale No. & For Impulse & For Pulse & For Impulse & For Pulse \\
0 & 1.0 & 0.916 & 1.0068 & 0.998 \\
1 & 0.527 & 3.29 & 0.210 & 0.442 \\
2 & 0.391 & 3.27 & 0.0037 & 0.231 \\
3 & 0.069 & 2.59 & 0.005 & 0.204 \\
\hline
\end{tabular}

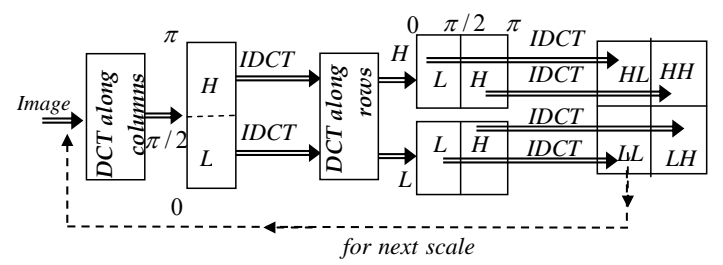

(a)
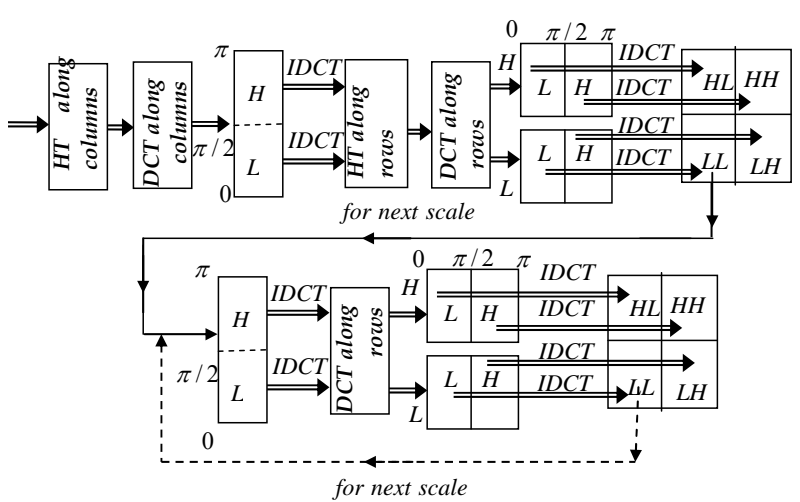

(b)

Figure 4. (a) Schematic for (a) the real part and (b) the imaginary part of 2D-ADCHWT.

its HT has to be considered. For this to start with, prior to along the columns of the image are taken and for these Hilbert transformed columns, the DCTs are taken (Figure 4(b)).

Further the DCT coefficients are grouped in to two groups and for each group, inverse DCT is applied to get the WT scales corresponding to the image columns. For each scale along the rows, the HTs are taken and for Hilbert transformed rows, the DCTs are taken. Again these DCT coefficients are grouped in to two groups and for each group inverse DCT is applied to get ADCHWT with scales HL, HH, LL and LH. As the HT has to be applied column and row wise only once, for higher scales, that is for splitting LL further, HT should not be applied and this is indicated in the Figure 3(b) which shows the absence column and row wise application of HT beyond LL scale. That is further LL scale decomposition is similar to that of the real part decomposition.

\section{Signal and Image Denoising Using ADCHWT}

Wavelet domain plays an important role in noise suppression. This is because, unlike removal of frequency components in FT based methods, here no (higher) frequency component is removed which results in smoothing of fast changes or edges. But in wavelet domain, noise suppression is done in time domain and hence no scale/frequency is removed unless it is totally not contributing to the signal. In wavelet denoising, in each scale, those values, which are below a certain threshold are made zero/modified and the signal is reconstructed with these modified scales. This is based on the assumption that the noise is distributed over all scales and their magnitudes will be small. But the problem with wavelet denoising is shift variant nature of WT (already been explained). In WT domain as the signal is reconstructed with modified decimated scale samples, there will be glitches as in between samples are removed especially at higher scales. The solution to this is to use shift invariant/ undecimated WT but this is at the expense of additional computational load. In view of this, the analytic WT, which provides shift invariance due to its reduced bandwidth by a factor of half, is an appropriate solution. Here in performing denoising, the threshold for each scale is found by considering the absolute values of ADCHWT coefficients. Further, the absolute values of ADCWT are compared with the estimated threshold to make a decision about whether a particular WT coefficient has to be retained/modified. This decision is applied to DCHWT (real domain) and the different modified scales are used for the signal reconstruction. In decision making not only the shift invariant nature of analytic WT but also its good envelope extraction property also contributes to it. Hence in some cases, the analytic WT based denoising performs better than those of shift invariant WT.

\section{Simulation Results}

The performance of the ADCHWT is shown for a discontinuous signal (SNR $=9 \mathrm{~dB}$ ) of 2048 points, speech segment $(\mathrm{SNR}=10 \mathrm{~dB})$ and an image $(\mathrm{SNR}=22 \mathrm{~dB})$. The noise associated with these is zero mean white Gaussian noise.

For the discontinuous signal, ADCHWT with 11 scales is considered and the denoising is done for lower 5 scales. For this, hard thresholding is done by using the standard deviation of the first scale scaled by a factor 6 as the threshold. The hard thresholding is given by

$$
f_{H T}(x)= \begin{cases}\operatorname{real}(x), & |x|>\mu \\ 0, & |x| \leq \mu\end{cases}
$$


where $f_{H T}(x)$ is the modified DCHWT for denoising, $\mu$ is the threshold and $x$ is the analytic WT coefficient value which is complex. ADCHWT showed improved performance as its $\mathrm{O} / \mathrm{P}$-SNR as $13.7 \mathrm{~dB}$ as against 13.1 dB for DCHWT and the glitches are of reduced magnitude compared to that of DCHWT (Figures 5(c) and (d)).

The speech, used is "Kaveriya Ugama Sthana Kodagu" sampled at 22050 Hzand quantized with 16 bits. A length of 1024 samples is used to generate frames with $50 \%$ overlap between successive frames. A 10 scale ADCHWT is considered for each frame. Further denoising by hard thresholding is done for lower 5 scales using the standard deviation of the first scale scaled by a factor 7 as the threshold. ADCHWT extracts the signal envelope well (Figures 6 (a), (c) and (d), a typical signal part is shown by encircling in Figure 6(d)). This results in a

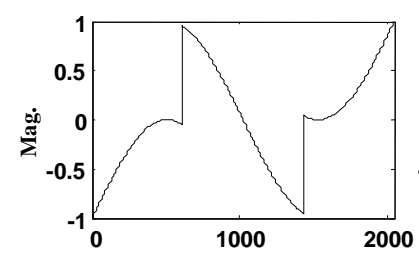

(a)

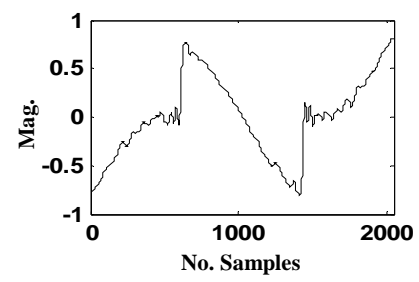

(c)

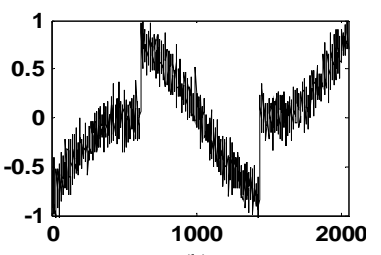

(b)

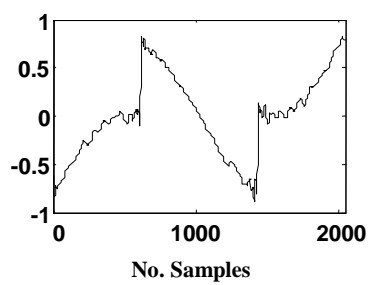

(d)
Figure 5. Denoising comparison for discontinuous signal (a) Clean, (b) Noisy (10 dB), (c) by DCHWT, (d) ADCHWT.

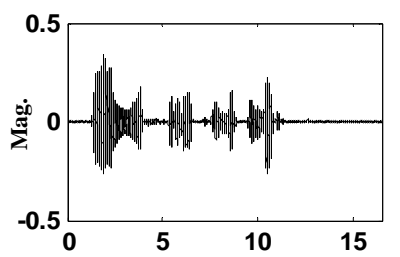

(a)

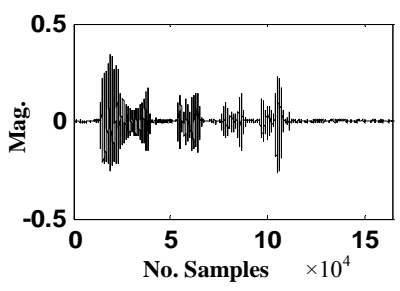

(c)

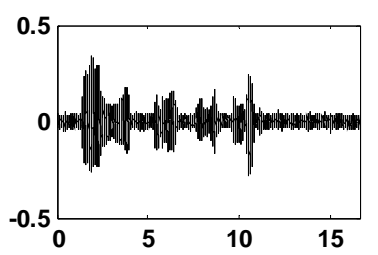

(b)

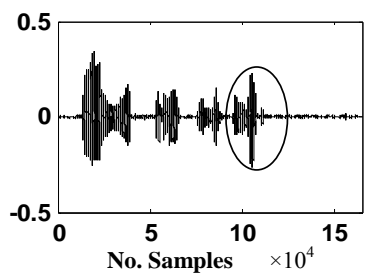

(d)
Figure 6. Denoising Comparison for speech (a) Clean, (b) Noisy (9dB), (c) DCHWT, (d) ADCHWT. better output. SNR by $4 \mathrm{~dB}$ compared to that of DCHWT. From hearing point of view, both sound somewhat similar, however DCHWT is having some glitches.

The image is decomposed into three scales with each scale consisting of four levels (LL, LH, HL and HH). So for three scales there are 12 levels. Denoising is carried out for all levels except scale-3, LL level assuming that it contains sufficiently large wavelet coefficients to represent the image. For image, a threshold value of 70 which corresponds to minimum error energy between the original and reconstructed image, is found experimentally as indicated in Table 2. Further, as seen from the Table 2, the optimum threshold point for DCHWT and ADCHWT occur at 60 and 70, respectively The output SNR with threshold value 60 is 18.7128 and 19.2958 for DCHWT and ADCHWT, respectively. But the output SNR for threshold value 70 is 18.3551 and 19.50 for DCHWT and ADCHWT, respectively and for ADCHWT, the output SNR is better by $1.2 \mathrm{~dB}$ compared to that of DCHWT. This also evident from Figures 7(a), (c) and (d) as the overall denoising is better for ADCHWT specially in bringing out the details.

\section{Conclusions}

A new dual tree Analytic Cosine Harmonic Wavelet transform was proposed. The analytic DCHWT was realized by applying DCHWT to the original signal and its Hilbert transform.

For both impulse and pulse input signals, its shift invariant property was found to be superior to that of DCHWT. Its application to noisy discontinuous signal, speech and image; indicated that due to its shift invariant and envelope preserving properties in deciding the modification of WT has resulted in a superior denoising performance than those of DCHWT. The real nature and the

Table 2. Threshold selection criteria for image denoising.

\begin{tabular}{ccc}
\hline Threshold & CHWT RMS Error & AHWT RMS Error \\
\hline 10 & 0.1859 & 0.1874 \\
20 & 0.1745 & 0.1818 \\
30 & 0.1538 & 0.1660 \\
40 & 0.1323 & 0.1424 \\
50 & 0.1188 & 0.1207 \\
60 & 0.1160 & 0.1084 \\
70 & $\mathbf{0 . 1 2 0 8}$ & $\mathbf{0 . 1 0 5 9}$ \\
80 & 0.1281 & 0.1092 \\
90 & 0.1363 & 0.1146 \\
100 & 0.1428 & 0.1208 \\
\hline
\end{tabular}




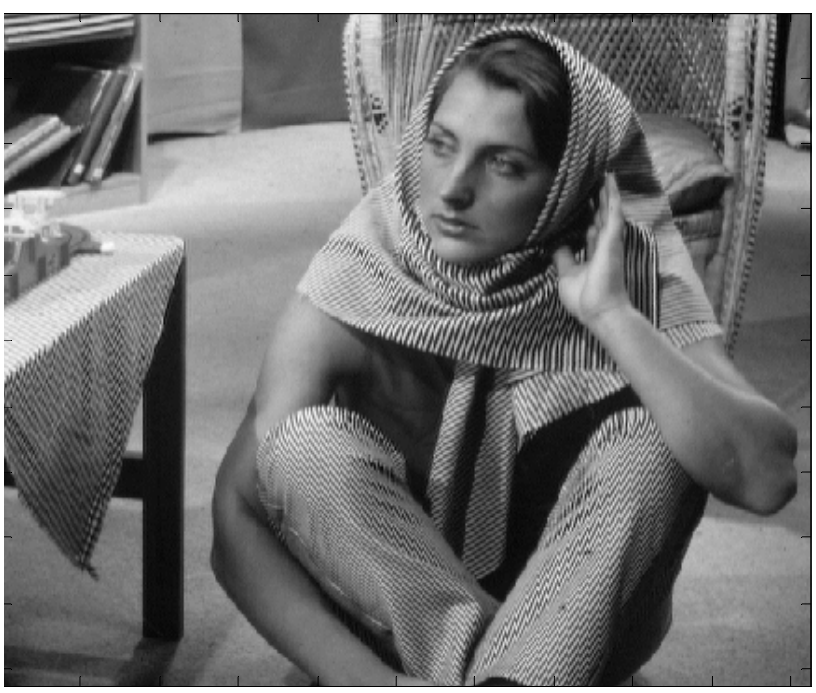

(a)

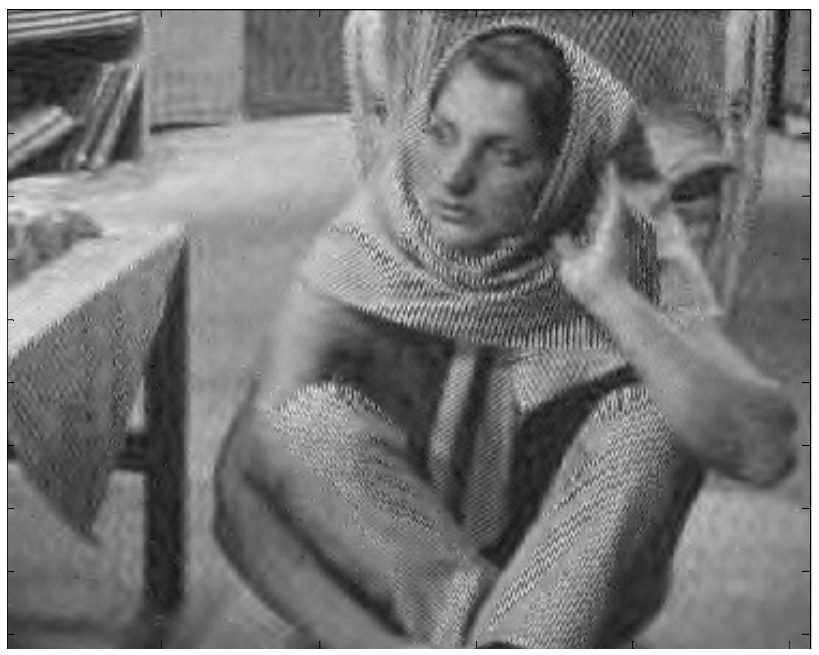

(c)

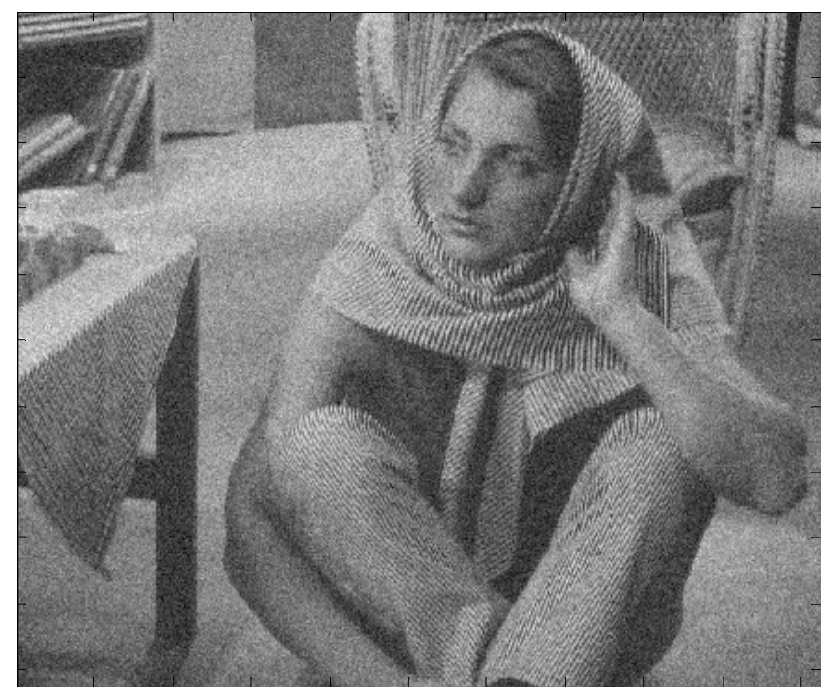

(b)

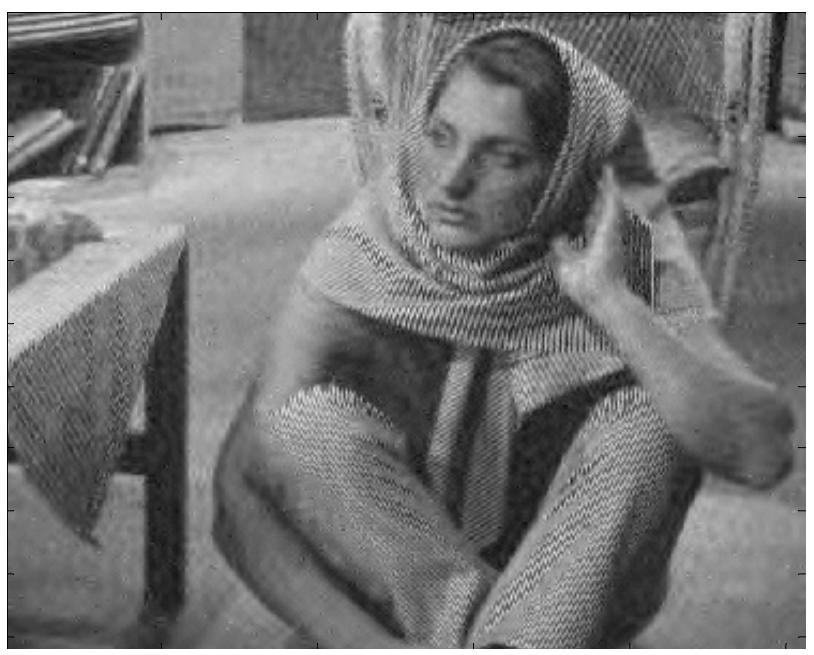

(d)

Figure 7. Comparison of DCHWT and ADCHWT for image with I/P - SNR = 22 dB. (a) Clean Image, (b) Noisy image, (c) DCHWT, (d) ADCHWT.

built in decimation and interpolation without any explicit filtering and delay compensation; makes the new algorithm simple and computationally efficient compared to other dual tree analytic algorithms.

\section{REFERENCES}

[1] I. W. Selesnick, R. G. Baraniuk and N. G. Kinsbury, "The Dual Tree Complex Wavelet Transform," IEEE Signal Processing Magazine, Vol. 22, No. 6, 2005, pp. 123-151. doi:10.1109/MSP.2005.1550194

[2] S. V. Narasimhan, A. R. Haripriya and B. K. Shreyamsha Kumar, "Improved Wigner-Ville Distribution Performance Based on DCT/DF Tharmonic Wavelet Transform and Modified Magnitude Group Delay," Signal Process- ing, Vol. 88, No. 1, 2008, pp. 1-18. doi:10.1016/j.sigpro.2007.06.013

[3] E. Hostalkova and A. Prochazka, "Complex Wavelet Transform in Biomedical Image Denoising," Proceedings of 15th Annual Conference Technical Computing Prague, 14 November, 2007, pp. 1-8.

[4] N. Terzija and W. Geisselhardt, "Digital Water Image Marking Using Complex Wavelet Transform," International Multimedia Conference, Proceedings of the Workshop on Multimedia and Security, Magdeburg, 20-21 September 2004, pp. 193-198.

[5] H. X. Lan, S. Q. Chen, T. S. Li and A. Hu, "A Digital Water Marking Algorithm Based on Dual Tree Complex Wavelet Transform," IEEE 9th International Conference for Young Computer Scientists, Hunan, 18-21 November 
2008, pp. 1488-1492.

[6] A. Jacquin, E. Causevic, R. John and J. Kovacevic, "Adaptive Complex Wavelet Based Filtering of EEG for Extraction of Evoked Potential Responses," International Conference on Acoustics, Speech and Signal Processing, Philadelphia, 18-23 March 2005, pp. 393-396.

[7] X. Zhu and J. Kim, "Application of Analytic Wavelet Transform to Analysis of Highly Impulsive Noises," Journal of Sound and Vibration, Vol. 294, No. 4-5, 2006, pp. 841-855. doi:10.1016/j.jsv.2005.12.034

[8] D. E. Newland, "Harmonic Wavelet Analysis," Proceedings of Royal Society-Series A, Vol. 444, 1993, pp. 203225.

[9] S. V. Narasimhan, M. Harish, A. R. Haripriya and N. Basumallick, "Discrete Cosine Harmonic Wavelet Transform and Its Application to Signal Compression and Subband Spectral Estimation Using Modified Group Delay," Signal, Image and Video Processing, Vol. 3, No. 1, 2009, pp. 85-99. doi:10.1007/s11760-008-0062-7

[10] N. Basumallick and S. V. Narasimhan, "A Discrete Cosine Adaptive Harmonic Wavelet Packet and Its Application to Signal Compression," Journal of Signal and Information Processing, Vol. 1, No. 1, 2010, pp. 63-76.

[11] S. V. Narasimhan and M. Harish, "A New Spectral Estimator Based on Discrete Cosine Transform and Modified Group Delay," Signal Processing, Vol. 86, No. 7, 2006, pp. 1586-1596. doi:10.1016/j.sigpro.2005.09.002

[12] S. V. Narasimhan and A. Adiga, "Shift Invariant Discrete Cosine Harmonic Wavelet Transform and Its Application to Denoising," IEEE INDICON, Bangalore, 6-8 September 2007.

[13] M. Shivamurti and S. V. Narasimhan, "Analytic Discrete Cosine Harmonic Wavelet Transform (ADCHWT) and Its Application to Signal/Image Denoising," IEEE International Conference on Signal Processing and Communication, Bangalore, 18-21 July 2010, pp. 1-5. 